\title{
Espacio literario relevante sobre la evaluación de la calidad del servicio: países de realización de los estudios, métodos de análisis, índices de fiabilidad, hipótesis y desafíos
}

\section{Relevant Literary Space about Service Quality Evaluation: Countries where the Studies are Performed, Analytical Methods, Reliability Assessments, Hypothesis and Future Research}

\author{
Pérez-Rave Jorge Iván \\ Departamento de Ingeniería Industrial, Gestión de la Calidad \\ Universidad de Antioquia, Medellín, Colombia \\ Correo:ejipr056@udea.edu.co,
}

\author{
Muñoz-Giraldo Leandro \\ Departamento de Ingeniería Industrial, Gestión de la Calidad \\ Universidad de Antioquia, Medellín, Colombia \\ Correo: investigagcalidad@udea.edu.co
}

Información del artículo: recibido: mayo de 2013, aceptado: julio de 2013

\section{Resumen}

Este artículo tiene como objetivo explorar el pensamiento vigente sobre el estudio de la calidad del servicio, a la luz de cinco variables. Se siguió la metodología de revisión sistemática de literatura en ingeniería (RSLI), con las etapas: identificar, describir, profundizar y divulgar. Luego del primer filtro de estudios con base en el mapa de delimitación, el control de calidad final consistió en verificar 257 documentos (90.3\% superó los criterios de inclusión). Según el análisis de representatividad del espacio literario relevante (ELR) se eligieron los 50 trabajos más posicionados (top 50), esto representó $4.2 \%$ de la población delimitada (1,019 documentos) y consolidó el $44.7 \%$ de las citas dadas al tema. China se erigió como el país con mayor presencia en el ELR, así como también los modelos de ecuaciones estructurales y el índice Alpha Cronbach; este último arrojó valores medios (0.87) muy superiores al referente tradicional de aceptación (0.7). En las hipótesis más aceptadas sobresalieron relaciones entre las variables/constructos: calidad, satisfacción, valor percibido e intenciones conductuales. Se identificaron seis categorías de desafíos futuros, donde dos de ellas fueron ampliar el alcance hacia otros contextos y profundizar en relaciones entre variables/constructos.

\section{Descriptores:}

- revisión sistemática de literatura en ingeniería

- RSLI

- calidad del servicio

- estado del arte

- revisión sistemática 


\begin{abstract}
This paper aims to explore the current thinking about service quality study, including five variables. We used the methodology of Systematic Literature Review in Engineering (RSLI) (steps: identifying, describing, deepening and publishing). After the first selection of documents based on the delimitation map, the final quality control consisted in checking 257 documents (90.3\% exceeded the inclusion criteria). According to the representativeness analisys, on the Relevant Literary Space, (ELR) the top 50 were chosen, which represented $4.2 \%$ of the population (1,019 documents) and consolidated $44.7 \%$ of the citations issued to the subject. China is the country with the largest presence in the ELR as well as Structural Equation Models; the Alpha Cronbach is the most used reliability index with a mean value of 0.87 , being higher compared to the traditional acceptance value (0.7). In most accepted hypotheses there are proved relationships among variables/constructs: quality, satisfaction, perceived value, and behavioural intentions. It identifies six categories of future research, two of them are to expand the scope of research to other contexts and deepen relations between variables/constructs.
\end{abstract}

\section{Keywords:}

- systematic literature review in engineering

- RSLI

- systematic review

- state of art

- service quality

\section{Introducción}

Este artículo tiene como objetivo posibilitar una mejor comprensión del pensamiento vigente sobre el estudio de la calidad del servicio, a la luz de cinco variables, entre ellas los desafíos futuros. Para ello se localiza y caracteriza un espacio literario relevante (ELR) sobre el tema, recurriendo a la metodología de revisión sistemática de literatura en ingeniería (RSLI) (Pérez, 2012), a fin de posibilitar reproducibilidad de los resultados, eficacia en la identificación de literatura posicionada y eficiencia a los investigadores o estudiantes en formación. En esa vía, la pregunta que guía el presente el trabajo es la siguiente: ¿qué características ha presentado la evaluación de la CS, como eje central, entre 2006 y 2011, en términos de: países de realización de los estudios, métodos de análisis, índices de fiabilidad de los instrumentos, hipótesis aceptadas y desafíos futuros?

A diferencia de la revisión tradicional (o narrativa), este trabajo, como podrá verse en el siguiente apartado, emplea métodos planeados, reproducibles y abiertos al escrutinio, que eliminan posibles sesgos de inclusión/ exclusión de estudios derivados de preferencias, inclinaciones o modelos mentales del "experto"; asimismo, se mitigan imprecisiones idiosincrásicas del revisor respecto a la interpretación de la abundante información narrativa, en el sentido de que se concluye a partir del procesamiento, en casos bajo meta-análisis, de la evidencia disponible, alusiva a las variables de interés y explícita directamente en estudios primarios. Para profundizar en diferencias entre la revisión tradicional y la sistemática véase Pérez (2012, pp. xvii).

La exploración del pensamiento vigente sobre el tema, a la luz de las cinco variables descritas, presenta implicaciones prácticas para inspirar trabajos venideros sobre la evaluación de la calidad del servicio, tanto a nivel de pregrado como posgrado e incluso para el medio empresarial. La caracterización al nivel de los países de realización de los estudios se incorporó con el fin de ubicar referentes internacionales y posibilitar alianzas y contrastes en materia de investigación en el tema; respecto a métodos de análisis, índices de fiabilidad e hipótesis, la razón fue aportar desde lo procedimental, de modo que se nutran los futuros proyectos, así como la enseñanza-aprendizaje sobre la evaluación de la calidad del servicio; los desafíos futuros no deben faltar en estudios de revisión, puesto que retratan vacíos en el conocimiento científico y, por lo mismo, posibilitan el avance en el tema.

\section{Materiales y métodos}

Se siguieron los procesos de la RSLI: identificar, describir, profundizar y divulgar; este último correspondiendo a pautas para la elaboración y socialización del manuscrito. A continuación se exponen los procedimientos seguidos:

Mapa de delimitación del espacio literario y controles de calidad: el término de búsqueda fue "service quality", teniendo como primer criterio de control de calidad el que los estudios pertenecieran a revistas indizadas en SciVerse Scopus, la mayor base de datos de resúmenes y citas de literatura revisada por expertos y fuentes Web de calidad a nivel mundial, según lo expresan diferentes centros de información alrededor del mundo (Biblioteca Universidad de Sevilla, 2013; Concytec, 2013; Biblioteca Eduardo Fernández Botero Universidad de Medellín; 2013), así como también numerosos trabajos, entre ellos: 
(Codina, 2005; Scimago, 2006; Canedo et al., 2010; Pérez, 2012). Los demás criterios de inclusión de estudios se precisan en el mapa expuesto en la figura 1.

El control de calidad, aparte de excluir los estudios que no cumplieron con el mapa de delimitación del espacio literario directamente bajo el algoritmo en Scopus, también consideró la verificación de dos criterios en una muestra representativa, según sugieren Pérez (2012) y Pérez y Jaramillo (2013). El primer criterio de verificación fue corroborar que los estudios fueran trabajos primarios, descartando cualquier estudio de revisión que posiblemente no se hubiera excluido en el filtro del algoritmo de búsqueda. El segundo criterio se debe a que cada estudio trate la calidad del servicio desde lo actitudinal del usuario (indicadores blandos), y no desde el tema QoS (quality of service), propio de áreas computacionales, el cual se enfoca en la continuidad en la transmisión de datos (indicadores duros). Con la fórmula de muestreo aleatorio simple para variables cualitativas el tamaño de estudios por verificar fue 257 documentos, a partir de los siguientes criterios: N (población): 1.129 documentos, 95\% de confianza, error máximo permisible de 0.05 , y p de 0.68 (proporción de documentos donde "service quality" es tratado desde la perspectiva de las actitudes de los usuarios [tema de interés] y que además, no son documentos tipo revisión). Este último parámetro fue supuesto con base en el número de documentos en la población que pertenecen al área de ciencias de la computación, por su cercanía con el tema QoS (359 documentos).

La verificación de los 257 documentos (título y resumen) constó de lo siguiente: un censo de las primeras 60 publicaciones que reportaron el mayor número de citas y verificación aleatoria para las restantes. Entre los 257 estudios verificados 25 no cumplieron con los criterios de inclusión (6 de ellos descartados en el censo), lo que permitió estimar $p$ experimental en 0.903 . Es decir, se estima que el $90.3 \%$ de la población de documentos ya filtrados mediante la ejecución del mapa de delimitación (figura 1) en Scopus, cumple con los criterios de verificación: 1,019 documentos.
Espacio literario relevante y su representatividad: para elegir el ELR entre la población de documentos que superaron los controles de calidad $(1,019)$, apoyados en Pérez y Jaramillo (2013) se recurrió a dos criterios más: número total de citas y los indicadores pCAT (porcentaje de citaciones atribuidas al top) y pDAT (porcentaje de documentos atribuidos al top). El número de citas se incluyó porque es una variable que alude la trascendencia o impacto que el estudio ha tenido en la comunidad y los dos indicadores expuestos se deben al supuesto, por analogía con el Principio de Pareto, de la separación entre lo "poco-vital" y lo "mucho-trivial" (Pérez, 2012). Al ordenar de mayor a menor número de citas, se reunieron cerca de 3,059 citas luego de haber efectuado el control de calidad. En la figura 2 se muestra el comportamiento del pCAT según el número de documentos a considerar como ELR (Top), junto con indicadores de significancia y ajuste (Statgraphics 5.1).

Fruto del análisis de representatividad del ELR se decidió abordar el top 50, el cual constituyó 4.9\% (pDAT; "poco") de la población de documentos delimitada $(1,019)$ y logró reunir $44.7 \%$ (pCAT: "vital") de la totalidad de citas dadas al tema bajo los criterios de búsqueda y control de calidad $(3,059)$. Considerando todo lo expuesto, en la tabla 1, por criterios de extensión, se presentan las 10 primeras posiciones del ELR (top 50) localizado.

Vale notar que el top 50 lo cerró Brown (2007) con 13 citas para un artículo publicado en Public Administration Review en 2007. La lista completa del ELR (top 50) puede solicitarse por correo al autor de correspondencia.

Descripción y caracterización del ELR: se leyó y sintetizó en forma narrativa cada estudio del ELR, a fin de extraer la información precisa sobre las variables de caracterización, ejecutando luego el control de calidad para anular: imprecisión, superfluo, sin coherencia/ progresión temática, lenguaje incomprensible, entre otros (Pérez, 2012, pp.51-53). Luego se agruparon los hallazgos narrativos en categorías de respuesta cerradas, que permitieran generar tabulaciones o conteos y adoptar una visión de totalidades para facilitar la

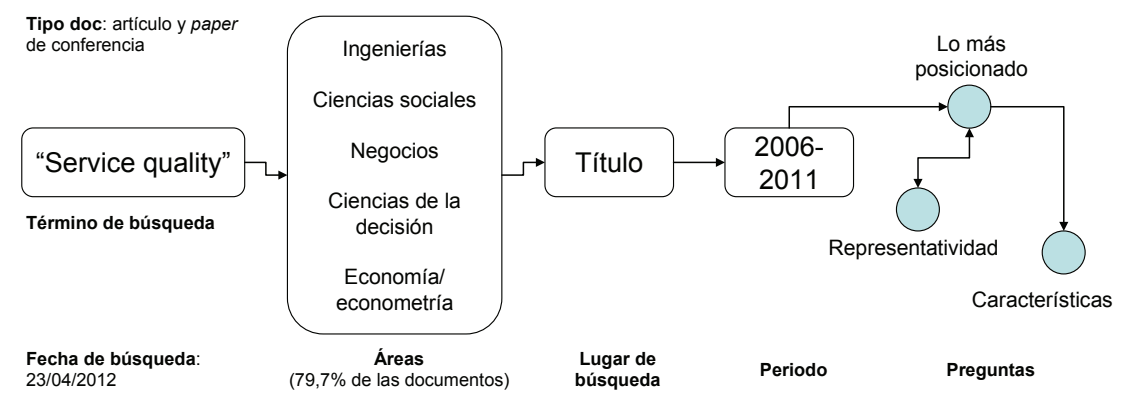

Figura 1. Mapa de delimitación del espacio literario (Pérez y Muñoz, 2012) 
DOI: https://doi.org/10.1016/S1405-7743(14)70356-7

Espacio literario relevante sobre la evaluación de la calidad del servicio: países de realización de los estudios, métodos de análisis, índices de ...

\begin{tabular}{|c|c|c|c|c|c|}
\hline Posición & Estudio & Título & Revista & Citas & Tabla 1. Diez primeras posiciones \\
\hline 1 & $\begin{array}{l}\text { Collier y } \\
\text { Bienstock (2006) }\end{array}$ & Measuring service quality in E-retailing & $\begin{array}{l}\text { Journal } \\
\text { of Service } \\
\text { Research }\end{array}$ & 118 & $\begin{array}{l}\text { de la calidad del servicio entre } \\
2006 \text { y } 2011 \text { (Pérez y Muñoz, } \\
\text { 2012) }\end{array}$ \\
\hline 2 & $\begin{array}{l}\text { Bauer et al. } \\
(2006)\end{array}$ & $\begin{array}{l}\text { eTransQual: A transaction process-based } \\
\text { approach for capturing service quality in } \\
\text { online shopping }\end{array}$ & $\begin{array}{l}\text { Journal of } \\
\text { Business } \\
\text { Research }\end{array}$ & 84 & \\
\hline 3 & $\begin{array}{l}\text { Cristobal et al. } \\
(2007)\end{array}$ & $\begin{array}{l}\text { Perceived e-service quality (PeSQ): } \\
\text { Measurement validation and effects on } \\
\text { consumer satisfaction and web site loyalty }\end{array}$ & $\begin{array}{l}\text { Managing } \\
\text { Service } \\
\text { Quality }\end{array}$ & 48 & \\
\hline 4 & $\begin{array}{l}\text { Olorunniwo et } \\
\text { al. (2006) }\end{array}$ & $\begin{array}{l}\text { Service quality, customer satisfaction, and } \\
\text { behavioral intentions in the service factory }\end{array}$ & $\begin{array}{l}\text { Journal of } \\
\text { Services } \\
\text { Marketing }\end{array}$ & 47 & \\
\hline 5 & Akbaba (2006) & $\begin{array}{l}\text { Measuring service quality in the hotel } \\
\text { industry: A study in a business hotel in } \\
\text { Turkey }\end{array}$ & $\begin{array}{l}\text { Int. Journal } \\
\text { of Hospitality } \\
\text { Management }\end{array}$ & 40 & \\
\hline 6 & $\begin{array}{l}\text { Wilkins et al. } \\
\text { (2007) }\end{array}$ & $\begin{array}{l}\text { Towards an understanding of total service } \\
\text { quality in hotels }\end{array}$ & $\begin{array}{l}\text { International } \\
\text { Journal of } \\
\text { Hospitality } \\
\text { Management }\end{array}$ & 36 & \\
\hline 6 & Ho y Lee (2007) & $\begin{array}{l}\text { The development of an e-travel service } \\
\text { quality scale }\end{array}$ & $\begin{array}{l}\text { Tourism } \\
\text { Management }\end{array}$ & 36 & \\
\hline 6 & $\begin{array}{l}\text { Dagger et al. } \\
\text { (2007) }\end{array}$ & $\begin{array}{l}\text { A hierarchical model of health service } \\
\text { quality: Scale development and } \\
\text { investigation of an integrated model }\end{array}$ & $\begin{array}{l}\text { Journal } \\
\text { of Service } \\
\text { Research }\end{array}$ & 36 & \\
\hline 9 & $\begin{array}{l}\text { González et al. } \\
\text { (2007) }\end{array}$ & $\begin{array}{l}\text { Assessing tourist behavioral intentions } \\
\text { through perceived service quality and } \\
\text { customer satisfaction }\end{array}$ & $\begin{array}{l}\text { Journal of } \\
\text { Business } \\
\text { Research }\end{array}$ & 35 & \\
\hline 10 & $\begin{array}{l}\text { Hsieh et al. } \\
(2008)\end{array}$ & $\begin{array}{l}\text { A service quality measurement } \\
\text { architecture for hot spring hotels in } \\
\text { Taiwan }\end{array}$ & $\begin{array}{c}\text { Tourism } \\
\text { Management }\end{array}$ & 34 & \\
\hline
\end{tabular}

Tabla 2. Ejemplo de extracto de constructos e índices de fiabilidad para e-service (Bauer et al., 2006)

\begin{tabular}{ll}
\hline Constructos evaluados & Índice de fiabilidad \\
\hline Calidad del servicio: & Alfa de Cronbach: \\
funcionalidad/diseño & $0.89(1), 0.84(2), 0.88$ (3), 0.83 \\
$\begin{array}{l}\text { (1) disfrute (2), proceso } \\
\text { (3), confiabilidad (4). }\end{array}$ & $(4), 0.85(5)$. \\
sensibilidad (5). & \\
\hline
\end{tabular}

Tabla 3. Extracto de las hipótesis planteadas por Collier y Bienstock (2006)

\begin{tabular}{lc}
\hline Hipótesis planteadas & Estatus \\
\hline $\begin{array}{l}\text { H1: Existe una relación positiva significativa } \\
\text { entre la dimensión de proceso y la } \\
\text { satisfacción. }\end{array}$ & Aceptada \\
$\begin{array}{l}\text { H5: Existe una relación positiva significativa } \\
\text { entre la dimensión de desenlace y las }\end{array}$ & Rechazada \\
$\begin{array}{l}\text { intensiones conductuales. } \\
\text { H8: Existe una relación positiva significativa } \\
\text { entre la satisfacción y las futuras intensiones } \\
\text { conductuales. }\end{array}$ & Aceptada \\
\hline
\end{tabular}

\section{Gráfico del Modelo Ajustado}

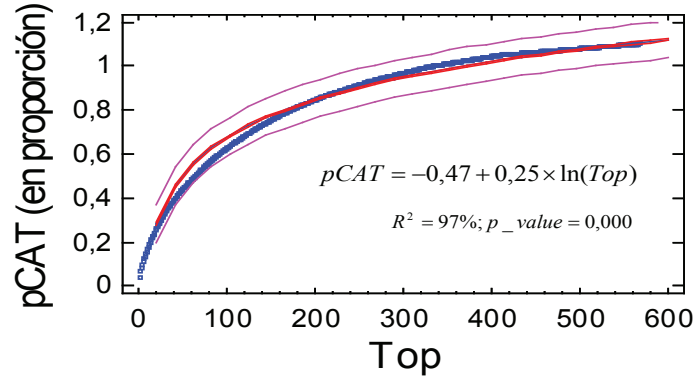

Figura 2. pCAT según el tamaño del top y el modelo que los relaciona 
comprensión global del objeto de profundización. Vea en la tabla 2 un ejemplo de la extracción y la tabulación de la información necesaria para abordar la variable "índices de fiabilidad".

Lo mismo se hizo para las variables: métodos de análisis e hipótesis aceptadas; en la tabla 3 se muestra un extracto de las evidencias para esta última.

Con base en la información alojada en la tabla 3 y en las demás elaboradas para este propósito (hipótesis), se desarrollaron diagramas que relacionaran los constructos o variables, bien se tratara de una relación causaefecto (se incorporaron flechas en una sola dirección) o de correlación (flechas bidireccionales). De esta manera fue posible tener una comprensión general de las hipótesis comúnmente aceptadas a partir de una muestra de $54 \%$ de los estudios en el top 50, la cual consistió en los trabajos pertenecientes a las cuatro tipologías de servicio más abordadas en el ELR: e-service, hotelería, aviación y bancos (Pérez y Muñoz, 2012). Esta muestra también se empleó para la variable desafíos futuros, resaltando que los estudios de la tipología aviación no precisó ninguno. A partir de esa muestra, se extrajeron categorías comunes que sintetizaran la redacción narrativa de cada estudio. Tanto a las tabulaciones como a los hallazgos obtenidos se les realizó control de calidad para ajustar posibles errores en tabulación, coherencia con la redacción narrativa y denominación de las categorías de respuesta.

\section{Resultados}

Países de realización de los estudios: en cuanto a la caracterización del lugar de realización de los estudios del ELR, en la tabla 4 se plasman los resultados. China encabeza la lista, participando con $20 \%$ de los documentos.

Métodos de análisis: primó el análisis de relaciones de causalidad, realizado a través de Modelos de Ecuaciones Estructurales (46\%), seguido del análisis de regresión $(24 \%)$, consolidando así $70 \%$ de los estudios del ELR; los demás porcentajes se distribuyeron en 14 categorías que consolidan desde 6 hasta $2 \%$, por mencionar algunas: diseño experimentos, lógica difusa y proceso analítico jerárquico AHP (Pérez y Muñoz, 2012).

Índices de fiabilidad: $68 \%$ de los estudios empleó el Alpha Cronbach, $11 \%$ fiabilidad compuesta y $21 \%$ restante usó ambos indicadores sobre una base de los 38 documentos que proporcionaron esta información (Pérez y Muñoz, 2012). En la tabla 5 se consolidan los resultados para los índices de fiablilidad Alpha Cronbach.

Los valores del coeficiente Alpha Cronbach oscilaron entre 0.65 (hotelería: Hu et al. 2009) y 0.99 (odontología: Ueltschy et al., 2007), con un promedio de 0.87 , obser-
Tabla 4. Países de origen de los servicios

\begin{tabular}{lcccc}
\hline $\begin{array}{l}\text { País de } \\
\text { realización }\end{array}$ & $\begin{array}{c}\text { Frec. } \\
\text { Absoluta }\end{array}$ & $\begin{array}{c}\text { Frec. Abs. } \\
\text { Acumulada }\end{array}$ & $\begin{array}{c}\text { Frec. } \\
\text { Relativa }\end{array}$ & $\begin{array}{c}\text { Frec. Rel. } \\
\text { Acum. }\end{array}$ \\
\hline China & 10 & 10 & $20 \%$ & $20 \%$ \\
España & 7 & 17 & $14 \%$ & $34 \%$ \\
USA & 7 & 24 & $14 \%$ & $48 \%$ \\
Australia & 6 & 30 & $12 \%$ & $60 \%$ \\
Reino Unido & 4 & 34 & $8 \%$ & $68 \%$ \\
$\begin{array}{l}\text { Varios } \\
\text { (multinacional) }\end{array}$ & 4 & 38 & $8 \%$ & $76 \%$ \\
Alemania & 3 & 41 & $6 \%$ & $82 \%$ \\
Grecia & 2 & 43 & $4 \%$ & $86 \%$ \\
Corea & 2 & 45 & $4 \%$ & $90 \%$ \\
Malasia & 2 & 47 & $4 \%$ & $94 \%$ \\
Escocia & 1 & 48 & $2 \%$ & $96 \%$ \\
$\begin{array}{l}\text { República de } \\
\text { Mauricio }\end{array}$ & 1 & 49 & $2 \%$ & $98 \%$ \\
Turquía & 1 & 50 & $2 \%$ & $100 \%$ \\
\hline
\end{tabular}

vando que en $75 \%$ de los casos el valor mínimo aceptado fue 0.82 (primer cuartil). Ello representa un referente retador para los futuros estudios, destacándose los servicios de odontología, salud y seguros con el primer cuartil superior a 0.90 .

Para el índice de fiabilidad compuesta, en promedio los valores se ubicaron en 0.83 , con mínimos y máximos de 0.56 y 0.96 , respectivamente. A nivel global, en $75 \%$ de los casos el valor mínimo fue 0.76 (tabla 6).

Hipótesis aceptadas: En las figuras 3 a 6 se consolidan las relaciones entre los elementos para los cuales se aceptaron las diferentes hipótesis probadas, según los tipos de servicios abordados. Los esquemas expuestos contienen un conjunto de pares de elementos conectados por medio de flechas. Algunas de ellas apuntan hacia dos elementos que integran una pareja determinada, haciendo referencia a la aceptación de hipótesis correlacionales (directas o inversas). Otras flechas apuntan hacia una sola dirección, significando relaciones causa $\rightarrow$ efecto.

Desafíos futuros: en la tabla 7, a modo de evidencia, se plasman los desafíos futuros precisos para cuatro de los estudios de la tipología e-service. Posteriormente se ofrecen los resultados consolidados para las seis categorías propuestas, fruto del análisis de patrones y simi- 
DOI: https://doi.org/10.1016/S1405-7743(14)70356-7

Espacio literario relevante sobre la evaluación de la calidad del servicio: países de realización de los estudios, métodos de análisis, índices de ...

Tabla 5. Estadísticas descriptivas para los coeficientes Alpha Cronbah

\begin{tabular}{|c|c|c|c|c|c|c|c|}
\hline Servicio & $\begin{array}{c}\text { Constructos con } \\
\text { Alpha* }\end{array}$ & Media & Desviación & Mínimo & Máximo & $\begin{array}{l}\text { Primer } \\
\text { cuartil }\end{array}$ & $\begin{array}{l}\text { Tercer } \\
\text { cuartil }\end{array}$ \\
\hline Aviación & 10 & 0.89 & 0.049 & 0.81 & 0.95 & 0.86 & 0.94 \\
\hline Bancos & 12 & 0.89 & 0.093 & 0.68 & 0.97 & 0.86 & 0.96 \\
\hline Call Center & 13 & 0.85 & 0.038 & 0.8 & 0.94 & 0.83 & 0.88 \\
\hline E-service & 50 & 0.87 & 0.057 & 0.72 & 0.95 & 0.83 & 0.91 \\
\hline Educación & 11 & 0.85 & 0.033 & 0.81 & 0.92 & 0.83 & 0.88 \\
\hline Esquí & 6 & 0.87 & 0.072 & 0.77 & 0.95 & 0.8 & 0.93 \\
\hline Hotelería & 30 & 0.83 & 0.074 & 0.65 & 0.96 & 0.78 & 0.89 \\
\hline Logística & 3 & 0.8 & 0.051 & 0.74 & 0.84 & 0.74 & 0.84 \\
\hline Multiservicios & 3 & 0.79 & 0.06 & 0.73 & 0.85 & 0.73 & 0.85 \\
\hline Odontología & 6 & 0.98 & 0.009 & 0.97 & 0.99 & 0.97 & 0.99 \\
\hline Restaurante & 4 & 0.78 & 0.039 & 0.74 & 0.82 & 0.75 & 0.82 \\
\hline Salud & 30 & 0.93 & 0.037 & 0.82 & 0.98 & 0.92 & 0.96 \\
\hline Seguros & 5 & 0.91 & 0.029 & 0.87 & 0.94 & 0.9 & 0.93 \\
\hline Spa & 2 & 0.87 & 0 & 0.87 & 0.87 & 0.87 & 0.87 \\
\hline Telefonía & 6 & 0.77 & 0.056 & 0.69 & 0.86 & 0.74 & 0.78 \\
\hline Global & 191 & 0.87 & 0.071 & 0.65 & 0.99 & 0.82 & 0.93 \\
\hline
\end{tabular}

*Se refiere a la cantidad de constructos de los tipos de servicios en cuestión, que arrojaron información precisa sobre el Alpha Cronbach. No debe confundirse con cantidad de estudios, ya que por ejemplo, un mismo estudio puede arrojar información para varios constructos (véase tabla 2).

litudes entre los desafíos con base en el procedimiento de acuerdo entre jueces.

Entre los estudios, 29 reportaron desafíos, con un promedio de 2 desafíos/estudio (desv. de 1). Se consolidaron 49 propuestas explícitas en la muestra (e-service, hotelería y bancos; aviación no reportó). La mayoría $(63 \%)$ se enfocó en sugerir ampliar el alcance hacia otros contextos y profundizar en relaciones entre facto- res o variables (entre los elementos tratados o adicionando otros). En la tabla 8 se presentan las frecuencias según cada categoría propuesta.

La distribución de las diferentes categorías de desafíos, según los tipos de servicio tratados, se plasma en la tabla 9. Nótese que a pesar de la variación de tipologías, prevalecen las dos categorías con mayor participación en el grupo consolidado (tabla 8).

Tabla 6. Estadísticas descriptivas para índices de fiabilidad compuesta

\begin{tabular}{lccccccc}
\hline Servicio & Frecuencia & Media & Desviación & Mínimo & Máximo & Primer cuartil & Tercer cuartil \\
\hline Aviación & 4 & 0.87 & 0.073 & 0.76 & 0.93 & 0.82 & 0.91 \\
Bancos & 7 & 0.80 & 0.157 & 0.56 & 0.96 & 0.65 & 0.94 \\
Biblioteca & 7 & 0.90 & 0.040 & 0.84 & 0.96 & 0.87 & 0.93 \\
Call center & 3 & 0.82 & 0.066 & 0.76 & 0.89 & 0.76 & 0.89 \\
E-service & 24 & 0.83 & 0.084 & 0.70 & 0.94 & 0.73 & 0.90 \\
Hotelería & 14 & 0.81 & 0.081 & 0.63 & 0.92 & 0.79 & 0.86 \\
Logística & 4 & 0.81 & 0.006 & 0.80 & 0.81 & 0.80 & 0.81 \\
Global & 63 & 0.83 & 0.088 & 0.56 & 0.96 & 0.76 & 0.90
\end{tabular}




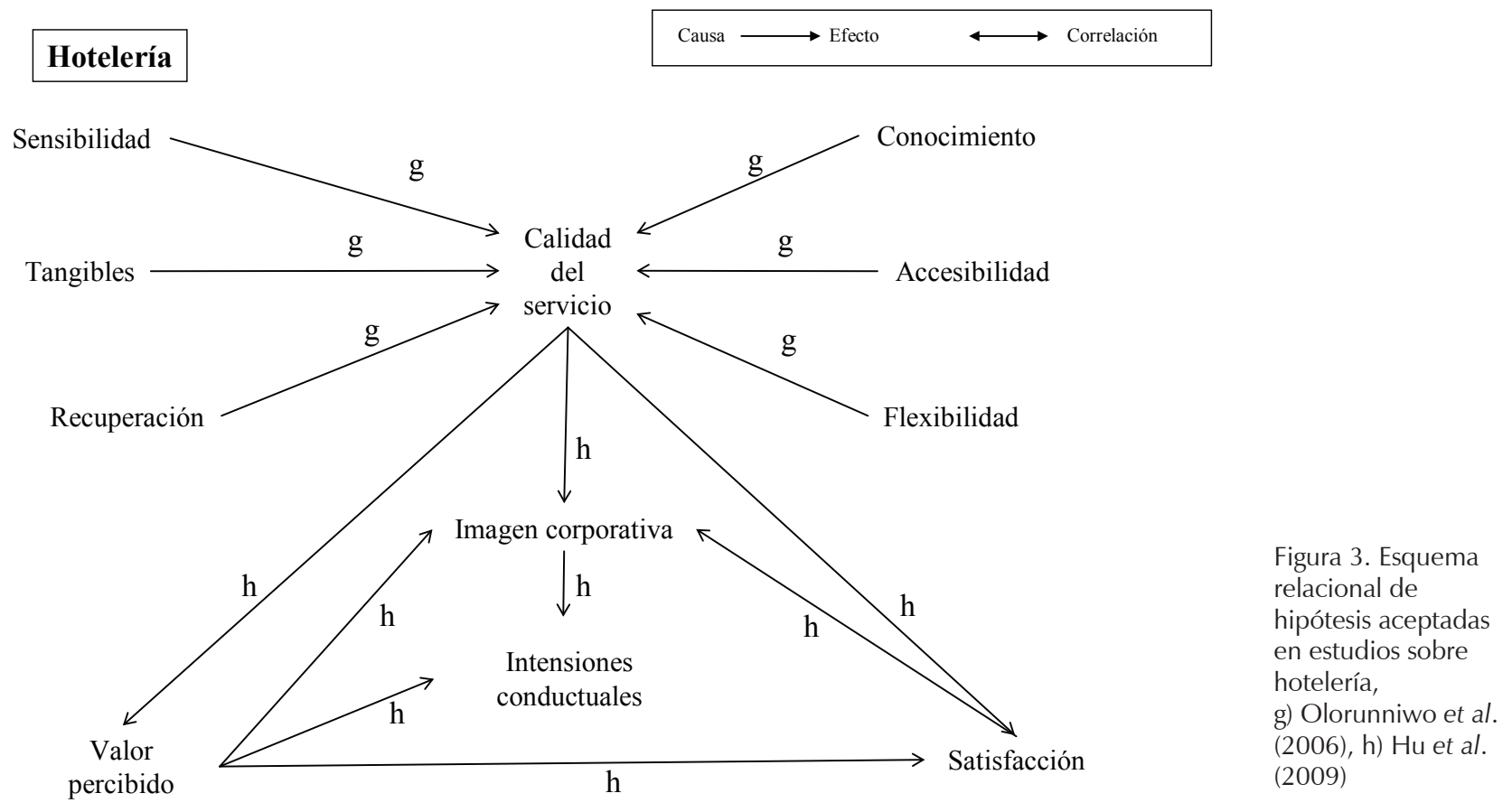

\section{e-service:}

Causa $\longrightarrow$ Efecto $\longleftrightarrow$ Correlación

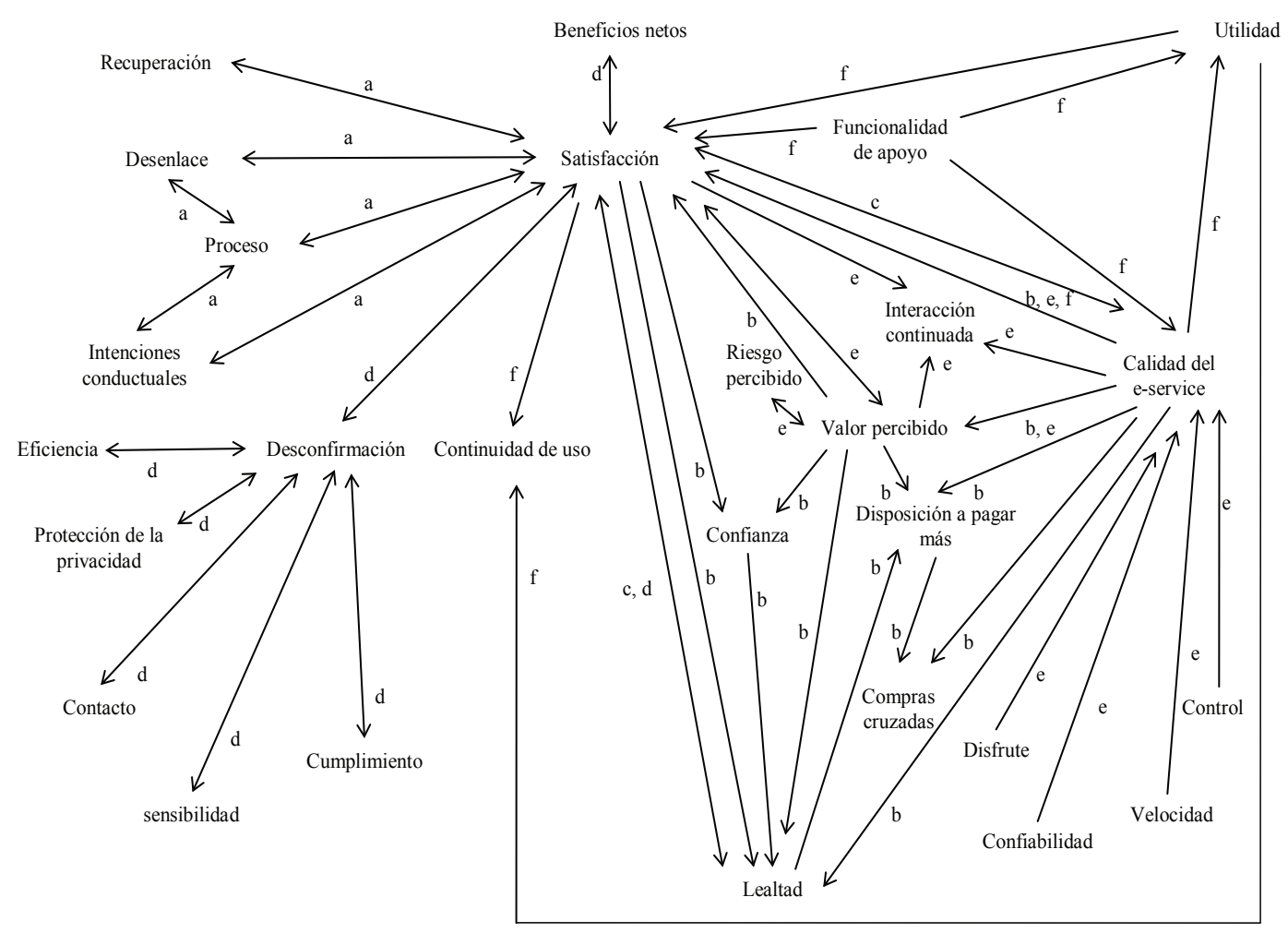

Figura 4. Esquema relacional de hipótesis aceptadas en estudios sobre e-service, a) Collier y Bienstock (2006), b) Fassnacht y Köse (2007), c) Cristobal et al. (2007), d) Yen y Lu (2008), e) Shamdasani et al. (2008), f) Cenfetelli et al. (2008) 

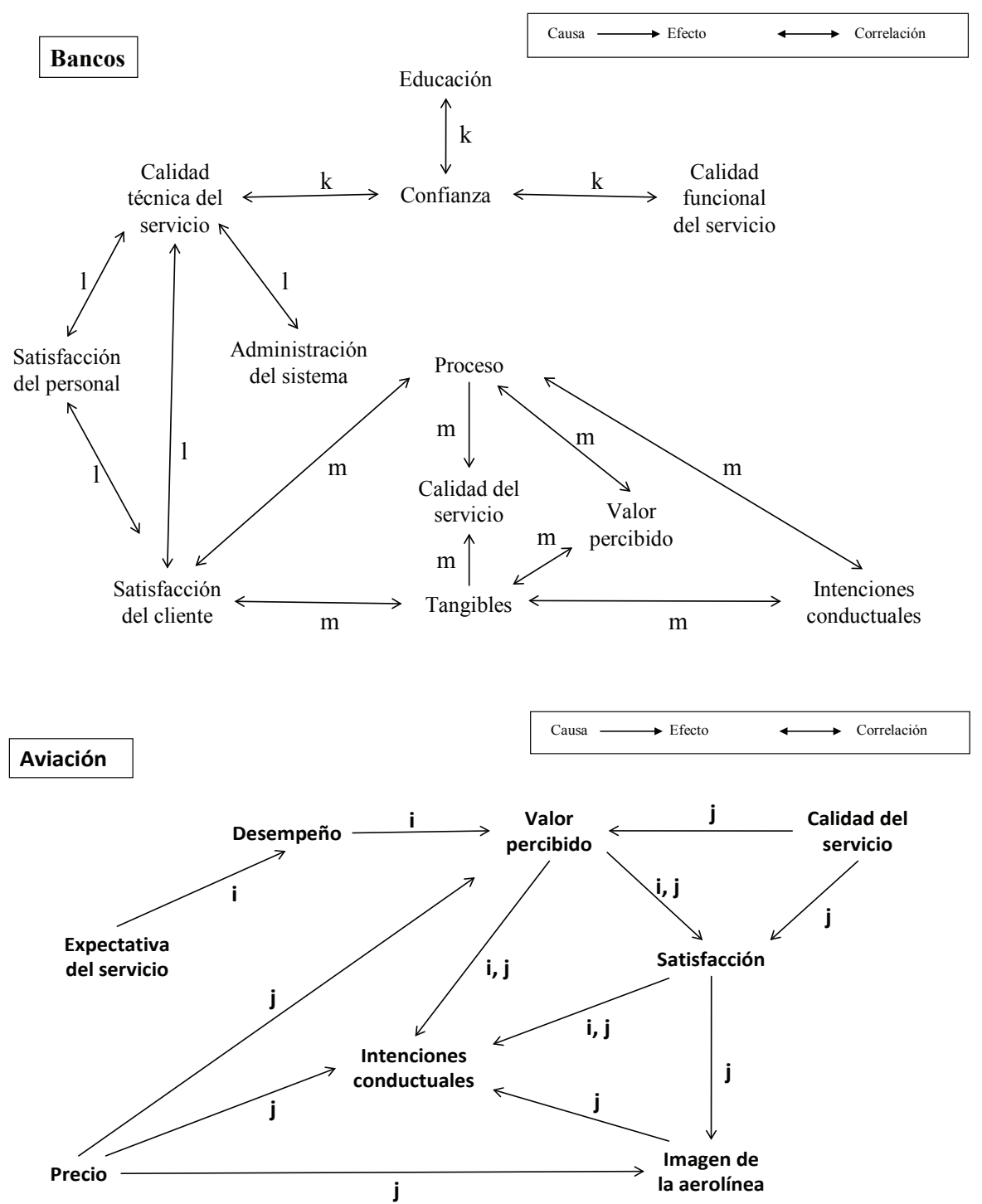

Figura 5. Esquema relacional de hipótesis aceptadas en estudios sobre bancos, k) Eisingerich y Bell (2008), I) Maddern et al. (2007), m) Yap y Sweeney (2007)

\section{Discusión}

En las fuentes bibliográficas examinadas del top, China se erige como el país en el que más se han realizado estudios sobre la calidad del servicio, con participación en 10 documentos ( $20 \%$ del top, vea la tabla 4$)$. Este interés evidente en la investigación de la calidad del servicio en el país asiático podría estar influenciado por el crecimiento económico acelerado del que ha gozado durante los últimos años y por la amplia inversión en investigación y desarrollo que, por ejemplo, en 2012 alcanzó nuevo record (mil millones de yuanes, de los cuales $74 \%$ provino del sector productivo) (CRI online, 2013). Es posible que, debido a la gran masa poblacional del país y, por ende, a una fuerte actividad comercial interna y externa, las organizaciones tengan que competir con vehemencia entre sí, producto de la elevada competencia en el mercado, haciendo del tema de la calidad del servicio una necesidad imperante para la investigación aplicada. En segunda instancia aparecen España y Estados Unidos como principales contribuyentes en investigación de la calidad del servicio, con una participación en 7 de los documentos (14\%) cada uno. En cuarto lugar se encuentra Australia con participación en 6 documentos ( $12 \%$ del top).

Con relación a los índices de fiabilidad, el coeficiente Alpha Cronbach mostró ser más utilizado que el de fiabilidad compuesta; es así que de los 38 estudios que arrojaron información sobre estos indicadores, solo $11 \%$ empleó en exclusiva el de fiabilidad compuesta, en tanto que $68 \%$ usó solo Alpha Cronbach y el 21\% restante recurrió a ambos indicadores. Sumado a lo ante- 
Tabla 7. Extracto de los desafíos futuros explícitos en los estudios del ELR para la tipología e-service

\begin{tabular}{|c|c|c|}
\hline Estudio & Categoría & Especificaciones \\
\hline \multirow{2}{*}{$\begin{array}{l}\text { Collier y Bienstock } \\
(2006)\end{array}$} & $\begin{array}{l}\text { Ampliar el alcance hacia otros } \\
\text { contextos }\end{array}$ & Diferentes organizaciones, industrias y contextos culturales \\
\hline & $\begin{array}{l}\text { Relaciones entre factores o } \\
\text { variables }\end{array}$ & $\begin{array}{l}\text { Satisfacción y futuras intenciones conductuales, satisfacción acumulada } \\
\text { y percepción general de la calidad }\end{array}$ \\
\hline \multirow[t]{2}{*}{ Ho y Lee (2007) } & $\begin{array}{l}\text { Profundizar en } \\
\text { particularidades de contextos } \\
\text { específicos }\end{array}$ & $\begin{array}{l}\text { Contenido de las dimensiones de calidad dependiendo de los } \\
\text { requerimientos específicos del servicio y las diferencias entre los } \\
\text { atributos de los sitios web }\end{array}$ \\
\hline & Estudios longitudinales & $\begin{array}{l}\text { Comparar las dimensiones de calidad por medio de estudios } \\
\text { longitudinales en el sitio web }\end{array}$ \\
\hline $\begin{array}{l}\text { Fassnacht y Köse } \\
\text { (2007) }\end{array}$ & $\begin{array}{l}\text { Adicionar complejidad a la } \\
\text { medición }\end{array}$ & $\begin{array}{l}\text { Efectos curvilíneos relacionales entre constructos; efectos moderadores } \\
\text { (interacciones) }\end{array}$ \\
\hline Tsai y Lu (2006) & Otras formas de evaluación & Servqual de tres columnas para medición de datos difusos \\
\hline
\end{tabular}

Tabla 8. Frecuencias para las categorías de desafíos futuros

\begin{tabular}{lcccc}
\hline Categorías de desafíos & Frec. Absoluta & Frec. Abs. Acum & Frec. Relativa & Frec. Rel. Acumulada \\
\hline Ampliar el alcance hacia otros contextos & 16 & 16 & $32.65 \%$ & $32.65 \%$ \\
Relaciones entre factores o variables & 15 & 31 & $30.61 \%$ & $63.27 \%$ \\
Adicionar complejidad a la medición & 5 & 36 & $10.2 \%$ & $73.47 \%$ \\
Otras formas de evaluación & 5 & 41 & $10.2 \%$ & $83.67 \%$ \\
Profundizar en particularidades de contextos & 4 & 45 & $8.16 \%$ & $91.84 \%$ \\
específicos & 4 & 49 & $8.16 \%$ & $100 \%$ \\
Estudios longitudinales & 4 & & \\
\hline
\end{tabular}

Tabla 9. Tabulación cruzada entre categorías de desafíos y tipología de servicios

\begin{tabular}{|c|c|c|c|c|c|c|c|}
\hline Tipología & $\begin{array}{l}\text { Ampliar } \\
\text { el alcance } \\
\text { hacia otros } \\
\text { contextos }\end{array}$ & $\begin{array}{l}\text { Relaciones } \\
\text { entre } \\
\text { factores o } \\
\text { variables }\end{array}$ & $\begin{array}{c}\text { Adicionar } \\
\text { complejidad a } \\
\text { la medición }\end{array}$ & $\begin{array}{l}\text { Otras formas } \\
\text { de evaluación }\end{array}$ & $\begin{array}{l}\text { Profundizar en } \\
\text { particularidades } \\
\text { de contextos } \\
\text { específicos }\end{array}$ & $\begin{array}{c}\text { Estudios } \\
\text { longitudinales }\end{array}$ & Total fila \\
\hline \multirow{2}{*}{ Bancos } & 4 & 4 & 0 & 0 & 2 & 1 & 11 \\
\hline & $36.36 \%$ & $36.36 \%$ & $0.00 \%$ & $0.00 \%$ & $18.18 \%$ & $9.09 \%$ & $22.45 \%$ \\
\hline \multirow{2}{*}{ Hotelería } & 7 & 5 & 2 & 4 & 0 & 0 & 18 \\
\hline & $38.89 \%$ & $27.78 \%$ & $11.11 \%$ & $22.22 \%$ & $0.00 \%$ & $0.00 \%$ & $36.73 \%$ \\
\hline \multirow{2}{*}{ E-service } & 5 & 6 & 3 & 1 & 2 & 3 & 20 \\
\hline & $25.00 \%$ & $30.00 \%$ & $15.00 \%$ & $5.00 \%$ & $10.00 \%$ & $15.00 \%$ & $40.82 \%$ \\
\hline \multirow{2}{*}{$\begin{array}{l}\text { Total } \\
\text { columna }\end{array}$} & 16 & 15 & 5 & 5 & 4 & 4 & 49 \\
\hline & $32.65 \%$ & $30.61 \%$ & $10.20 \%$ & $10.20 \%$ & $8.16 \%$ & $8.16 \%$ & $100.00 \%$ \\
\hline
\end{tabular}

rior, comparando los valores de estos dos indicadores, los referentes para el Alpha Cronbach son más altos que para el de fiabilidad compuesta (el primer cuartil para el Alpha Cronbach es 0.82, en tanto que para el de fiabilidad compuesta es 0.76 ).

Respecto a las hipótesis más aceptadas en el ELR, las figuras 3 a 6 facilitan deducir las relaciones más comúnmente aceptadas. En la figura 7 se muestran tales relaciones, donde la mayoría de ellas es de tipo causa $\rightarrow$ efecto. Observe que en los estudios sobre bancos no se reflejó este comportamiento; sin embargo, al extender los análisis hacia otros estudios del top 50 (restaurante, Spa, logística, seguros) estas mismas relaciones estuvieron presentes.

Pasando a los desafíos futuros, sobresale la necesidad de ampliar el alcance de las investigaciones, independiente de la tipología del servicio; es así que los estudios recomendaron aumentar el tamaño de la muestra, abarcar otros tipos de servicio, países, poblaciones, culturas, industrias, etcétera, a fin de analizar la 
posibilidad de generalizar los resultados obtenidos; algunos de los trabajos que sugieren este desafío son: Collier y Bienstock (2006). Cenfetelli et al. (2008), Tseng (2009) y Glaveli et al. (2006). Respecto a las relaciones entre factores o variables (Eisingerich y Bell, 2008; Yap y Sweeney, 2007; Albacete et al., 2007; entre otros) se hicieron evidentes varias situaciones, algunas clásicas que abordan dimensiones de calidad, satisfacción, calidad percibida e intenciones conductuales, pero también otras que infieren mayor complejidad en los estudios y ameritan una visión particularizada en fenómenos del usuario, así como una perspectiva global que analice el entorno que rodea al servicio.

La categoría de adicionar complejidad a la medición ocupó, junto con la de otras formas de evaluación, el tercer lugar en lo más sugerido (10.2\% cada una). Los estudios recomendaron considerar mediciones que traspasaran relaciones clásicas, profundizando en efectos curvilíneos relacionales entre constructos y efectos moderadores (interacciones) (Fassnacht y Köse, 2007); esto va en sincronía con el notable uso de los modelos de ecuaciones estructurales ( $46 \%$ del ELR) para estudiar relaciones de causalidad. También se sugieren métodos para abordar la interdependencia (por ejemplo, proceso de red analítico) (Hsieh et al., 2008), así como otros para impactar desde la misma estructura de medición (multi-jerárquica o multicriterio que considere efectos relacionales e interactivos) (Glaveli et al., 2006) y hasta evaluar la calidad del servicio teniendo en cuenta la incertidumbre de los términos lingüísticos en trabajos con lógica difusa (Tsai y Lu, 2006). Sobre las otras formas de evaluación, se hace referencia a la necesidad de llevar a cabo las mediciones de expectativas y percepciones en instantes diferentes (Akbaba, 2006); las expectativas antes del consumo y las percepciones posteriores al mismo. También se explicita incrementar el número de ítems para la caracterización de las intenciones conductuales y el valor percibido. Del mismo modo, en el caso de estudios con lógica difusa, se sugiere emplear el Servqual de tres columnas para medición de datos difusos (Tsai y Lu, 2006).

Las últimas dos posiciones las ocuparon las categorías profundizar en particularidades contextuales y realizar estudios longitudinales; algunos de ellos son: Ho y Lee (2007) y Yi y Gong (2008). Las particularidades tienen que ver con desarrollar conceptos específicos para contextos particulares o con profundizar en las funciones del servicio o las dimensiones de calidad más relevantes dependiendo de un escenario específico. Los estudios longitudinales hacen presencia ante la necesidad de mejor comprensión de la dinámica de la calidad o de variables asociadas.
De manera específica, para el caso de los desafíos futuros de los bancos (Yen y Lu, 2008; Eisingerich y Bell, 2008; entre otros) se precisó la necesidad de estudiar diversas relaciones bajo las siguientes variables o factores: educación del cliente, intenciones de recompra, disponibilidad de tiempo del usuario, costo percibido de adquirir técnicas de inversión, percepciones sobre aspectos tangibles, intenciones conductuales, reacciones que el cliente manifiesta luego de introducir mejoras al sistema, gasto en la calidad del servicio, objetivos financieros como la rentabilidad y el ROI, características del medio ambiente que rodea al servicio y la gestación de la calidad percibida del servicio.

Para el caso de hotelería (Olorunniwo et al., 2006; Akbaba, 2006; entre otros), las relaciones requeridas pueden resumirse en el estudio de las variables: satisfacción, intenciones conductuales, tipología del servicio, calidad del servicio, valor percibido, rentabilidad de las organizaciones, otras variables de desempeño de las firmas, contexto internacional (atributos relacionados con la influencia de las políticas estatales), cohibición y estimulación de las prácticas gerenciales para el mejoramiento de la calidad en el turismo, administración de las dimensiones de calidad del servicio en el alojamiento rural, niveles de fiabilidad y satisfacción de los turistas, comunicación boca a boca online, retroalimentación, toma de decisiones de los usuarios y brechas entre percepciones y expectativas.

En cuanto el e-service (Bauer et al., 2006; Fassnacht y Köse, 2007; por mencionar algunos), se explicitan: satisfacción, futuras intenciones conductuales, satisfacción acumulada, percepción general de la calidad, innova-

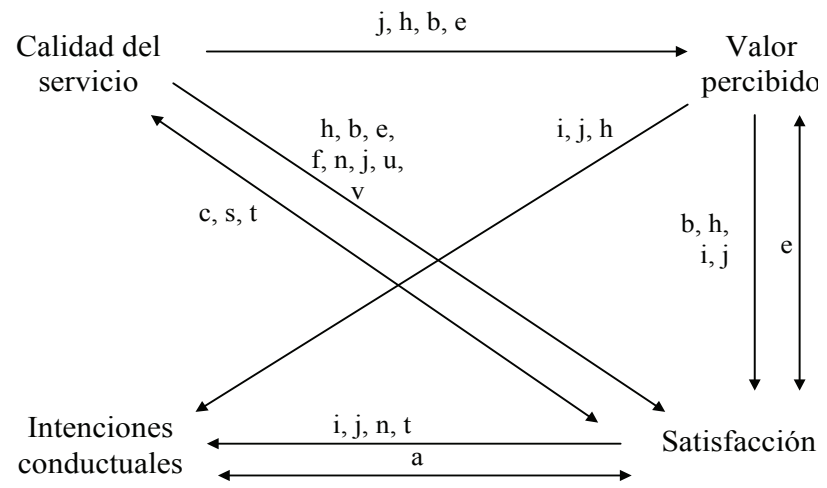

Figura 7. Constructos para los que más se aceptó algún tipo de relación en el ELR, aviación: i) Chen (2008), j) Park et al. (2006), hotelería: h) Hu et al. (2009), E-service: a) Collier y Bienstock (2006), b) Fassnacht y Köse (2007), c) Cristobal et al. (2007), e) Shamdasani et al. (2008), f) Cenfetelli et al. (2008), salud: n) Dagger et al. (2007), otros: s) Hau-siu et al. (2007), t) González et al. (2007), u) Saura et al. (2008), v) Tsoukatos y Rand (2006) 
ción, orientación al mercado, confianza, comportamientos en el usuario mucho más específicos, características del diseño de los mecanismos de subasta, factores de marketing, dimensiones de tipo económico (incremento del precio, etcétera), intenciones de recompra, diferencias demográficas, calidad del servicio, valor percibido, lealtad. Esto entra en consonancia con megatendencias como la mercadotecnia personalizada que alerta sobre la pérdida de vigencia de la producción en masa y sobre la necesidad de una interacción directa y bidireccional entre consumidores y organizaciones, con apoyo de TIC, servicios financieros y logísticos para incrementar el valor agregado en los grupos de interés (Juárez y González, 2009).

Ante los desafíos explícitos para cada tipología descrita, puede deducirse mayor complejidad y necesidad de esfuerzos por entender la calidad del servicio a la luz de: 1) el actuar del usuario ante variedad de situaciones o sentir; 2) visión en el contexto; es decir, no solo mirar el "árbol", sino también el "bosque" y las relaciones entre ellos (medio ambiente, políticas estatales,...); 3) reunir evidencia objetiva sobre las relaciones causales entre los medios o agentes facilitadores y los resultados empresariales, sobre todo de tipo financiero, para un mejor direccionamiento de las firmas ante las limitaciones de recursos.

Considerando los desafíos futuros en las tipologías de servicio (tabla 9), se corrobora, en cada caso, la supremacía de las dos categorías más sugeridas a nivel general (ampliar el alcance y profundizar en las relaciones entre factores o variables); sin embargo, en el caso de los bancos se destaca el profundizar en particularidades como: estudiar con mayor profundidad y complejidad el constructo educación del cliente, así como desafiar y mejorar el concepto de "zona de tolerancia", llevándolo también a otros servicios, como educación y salud. En hotelería cobran relevancia otras formas de evaluación de la calidad del servicio, básicamente empleando diferentes momentos de evaluación, primero la medición de expectativas y después, en un instante diferente, la de percepciones. Asimismo, incrementar el número de ítems para la caracterización de las intenciones conductuales y el valor percibido. En e-service se destaca adicionar complejidad a la medición, básicamente con relaciones no lineales, efectos moderadores, incertidumbre en términos lingüísticos (lógica difusa) y uso de enfoque multicriterio. También, con el mismo porcentaje $(15 \%)$, trascender estudios de corte trasversal para entender las dinámicas de la calidad.

A fin de explorar, en estudios recientes, la prevalencia de los hallazgos deducidos, se ejecutó el mismo mapa de delimitación del espacio de búsqueda que se presentó en la figura 1, con la diferencia de que esta vez el periodo se delimitó a 2013. En total se encontraron 62 publicaciones, tal como se evidencia en la figura 8 , de los cuales se espera, según lo estimado en el presente estudio, que $90.3 \%$ (56 estudios) cumplan con los criterios de interés (vea el apartado de materiales y métodos, sección mapa de delimitación del espacio literario y controles de calidad).

Luego de ello, con el fin de prevenir sesgar la inclusión/exclusión de estudios, se seleccionaron los 10 primeros trabajos que ofreció Scopus $(17.8 \%$ de la población delimitada) y, con base en ellos, prosiguió la exploración. De nuevo China participó notablemente en el grupo de estudios explorados (Pornpitakpan y Han, 2013; Ayeh y Chen, 2013; Lee et al., 2013). También se encontraron esfuerzos por ampliar el alcance de las investigaciones hacia otros contextos, independiente de la tipología de servicios, como se identificó en el ELR; por ejemplo, Oyewole (2013) enfocó la evaluación de la calidad del servicio haciendo hincapié en consumidores afroamericanos (características socioeconómicas y demográficas) manifestando que existen pocos estudios sobre su actitud y comportamiento hacia el consumo, en este caso en restaurantes tipo buffet. Lee, et al. (2013) dirigen el estudio hacia puertos, incorporando el punto de vista de las compañías navieras y señalando que es un estudio pionero. Ayeh y Chen (2013) reflejan esfuerzos por entender diversidad de sectores: agencias de hotel, de venta al por menor y de viajes en Hong Kong. Pornpitakpan y Han (2013) centran su interés en la diversidad de culturas: Singapur y Americana, y Jasinskas et al. (2013) buscan entender y aportar a la calidad de centros de fitness desde el punto de vista del Servqual, así como Akter et al. (2013) se orientan a servicios de salud móvil. Varios trabajos también estudian la calidad del servicio considerando particularidades de los usuarios, tales como: Oyewole (2013) (sexo, edad, ingresos económicos). Ayeh y Chen (2013) (asiáticos y no asiáticos), así como otros aportaron dimensiones de calidad para contextos específicos (Lee et al., 2013) y probaron relaciones con variables clásicas como satisfacción y valor percibido, intenciones conductuales, entre otras (Senić y Marinković. 2013; Oyewole (2013); Akter et al., 2013).

Otro aspecto por señalar es la coherencia con retos metodológicos (complejidad y otras formas de evaluación), entre los estudios al respecto se encuentran: Pornpitakpan y Han (2013) (experimentos transculturales: diseño factorial). Lee et al. (2013) (Proceso de análisis jerárquico, análisis factorial confirmatorio), Ayeh y Chen (2013) (análisis importancia-rendimiento), Duan et al. (2013) (técnica de análisis de sentimiento), Akter et al. (2013) (modelo multidimensional jerárquico) y el in- 


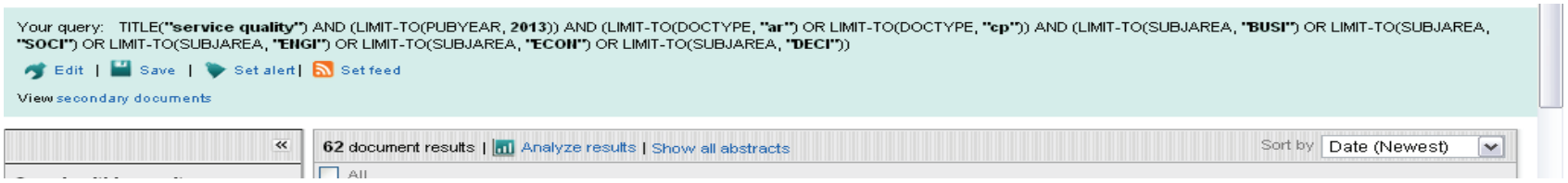

Figura 8. Algoritmo de búsqueda en Scopus para estudios recientes

terés por aportar nuevas medidas de la calidad (Lan, 2013; Lee et al., 2013; Akter, et al., 2013).

Aparte de lo realizado por los estudios recientes descritos, las agrupaciones propuestas en el presente artículo (véase tabla 8) también cobijan los desafíos futuros que estos plasman de forma explícita. Por ejemplo, Oyewole (2013) señala la necesidad de mayor estudio de la población específica abordada (afroamericana) incorporando: gasto mensual, comidas determinadas, contenido nutricional de los alimentos y razones de comportamientos particulares, lo cual se enmarca en la categoría "profundizar en particularidades de contextos específicos". Akter et al., (2013), refiriéndose a otra población, se suman a esa categoría agregando variables sociodemográficas (ingresos, educación, género, etcétera) y constructos situacionales; además de que motivan a replicar el estudio en otros países y contextos de servicios (otras de las categorías propuestas) y señalan la necesidad de comprender la dinámica de la calidad mediante mediciones longitudinales. Pornpitakpan y Han (2013) igualmente precisan como desafíos futuros la necesidad de ampliar el alcance hacia otros contextos, estudiar los efectos de diversas particularidades contextuales y profundizar en relaciones entre factores o variables (cantidad de compras, tiempo en la tienda, atractivo de los productos, relación calidad-precio y búsqueda de otras variables que puedan influir en la compra por impulso).

\section{Conclusiones}

Este artículo brinda hallazgos en cuanto al estudio de la calidad del servicio, provenientes de métodos de búsqueda, depuración y análisis, planeados, estructurados, libres de sesgos de inclusión/exclusión de estudios y de juicios personales del revisor, entre otras características. La profundización en el top 50 de estudios más posicionados sobre el tema llevó a identificar los países con mayor reporte de trabajos en el ELR, así como los índices de fiabilidad empleados y su descripción estadística. Esto último reflejó un nuevo referente para las futuras investigaciones respecto a la fiabilidad de los instrumentos de medición, ya que tradicionalmente se han aceptado valores superiores a 0.7 (Alpha Cronbach), pero los presentes hallazgos evidenciaron un desplaza- miento alrededor de 0.87 para estar a la altura de los trabajos más citados en el tema.

Del mismo modo, se encontró que las sugerencias de investigación apuntan, a futuro, a trascender los enfoques correlacionales, cobrando relevancia las relaciones causales mediante los modelos de ecuaciones estructurales, considerando no solo dimensiones de calidad y variables clásicas como satisfacción, intenciones conductuales, valor y calidad percibida, sino también relaciones de estas con aspectos característicos del usuario (educación,...) así como del contexto que rodea al servicio (medio ambiente, políticas de gobierno,...) y de variables clave del desempeño empresarial (rentabilidad,...).

Profundizar en estos desafíos futuros amerita, además, trascender los aportes metodológicos convencionales, llamando la atención sobre la pertinencia de ir más allá de modelos de relaciones lineales, de supuestos de certidumbre e independencia, y considerar efectos moderadores entre los elementos del modelo, posibles relaciones jerárquicas, incertidumbre de los parámetros, dinámicas de la calidad (estudios longitudinales). También se precisa la necesidad de estudiar posibilidades de generalización de los resultados o modelos (muestras más representativas, otros tipos de poblaciones, tipologías de servicio,...) y de identificar diferencias contextuales significativas que ameriten tratamientos personalizados en determinados objetos de intervención. Es así como los futuros estudios deberían contemplar como mínimo uno o más de los hallazgos expuestos en este artículo, a fin de agregar más valor a las diversas preguntas que se deriven de lo expuesto.

\section{Referencias}

Akbaba A. Measuring Service Quality in the Hotel Industry: A Study in a Business Hotel in Turkey. International Journal of Hospitality Management, volumen 25 (número 2), 2006: 170-192.

Akter S., D'Ambra J., Ray P. Development and Validation of an Instrument to Measure User perceived Service Quality of mHealth. Information and Management, volumen 50 (número 4), 2013: 181-195.

Albacete C., Fuentes M., Lloréns J. Service Quality Measurement in Rural Accommodation. Annals of Tourism Research, volumen 34 (número 1), 2007: 45-65.

Ayeh J. y Chen R. 'How's the service?' A Study of Service Quality Perceptions Across Sectors and Source Markets. International Journal of Tourism Research, volumen 15 (número 3), 2013: 241-260. 
Bauer H., Falk T., Hammerschmidt M. E-TransQual: A Transaction Process-Based Approach for Capturing Service Quality in Online Shopping. Journal of Business Research, volumen 59 (número 7), 2006: 866-875.

Biblioteca Eduardo Fernández Botero. Universidad de Medellín [Fecha de consulta: 13 de mayo de 2013] [en línea]. Disponible en: http://www.udem.edu.co/index.php/2012-11-23-20-25-35/ revistas-electronicas

Biblioteca Universidad de Sevilla [fecha de consulta: 13 de mayo de 2013] [en línea]. Disponible en: http://bib.us.es/Soportenews/news/bdd_marzo_scopus-ides-idweb.html

Briggs S., Sutherland J., Drummond S. Are Hotels Serving Quality? An Exploratory Study of Service Quality in the Scottish Hotel Sector. Tourism Management, volumen 28 (número 4), 2007: 1006-1019.

Brown T. Coercion Versus Choice: Citizen Evaluations of Public Service Quality Across Methods of Consumption. Public Administration Review, volumen 67 (número 3), 2007: 559-572.

Canedo R., Rodriguez R., Montejo M. Scopus: la mayor base de datos de literatura científica arbitrada al alcance de los países subdesarrollados. ACIMED [en linea] volumen 21 (número 3), 2010: 270-282.

Cenfetelli R., Benbasat I., Al-Natour S. Addressing the what and how of Online Services: Positioning Supporting-Services Functionality and Service Quality for Business-to-Consumer Success. Information Systems Research, volumen 19 (número 2), 2008: 161-181.

Chen C. Investigating Structural Relationships between Service Quality, Perceived Value, Satisfaction, and Behavioral Intentions for Air Passengers: Evidence from Taiwan. Transportation Research Part A: Policy and Practice, volumen 42 (número 4), 2008: 709-717.

Codina L. Scopus: el mayor navegador científico de la web. El profesional de la información, volumen 14 (número 1), 2005: 44-49.

Collier J. y Bienstock C. Measuring Service Quality in E-Retailing. Journal of Service Research, volumen 8 (número 3), 2006: 260-275.

Concytec. Recuperado [en línea] [fecha de consulta: 13 de mayo de 2013]. Disponible en: http://bvcyt.concytec.gob.pe/php/level.php?lang=es\&component $=32 \&$ item $=45$

CRI [en línea] [fecha de consulta: 11 de mayo de 2013]. Disponible en: http://espanol.cri.cn/782/2013/03/02/1s271754.htm

Cristoba E., Flavián C., Guinalíu M. Perceived E-Service Quality (PeSQ): Measurement Validation and Effects on Consumer Satisfaction and Web Site Loyalty. Managing Service Quality, volumen 17 (número 3), 2007: 317-340.

Dagger T., Sweeney J., Johnson, L. A Hierarchical Model of Health Service Quality: Scale Development and Investigation of an Integrated Model. Journal of Service Research, volumen 10 (número 2), 2007: 123-142.

Duan W., Cao Q., Yu Y., Levy S. Mining Online User-Generated Content: Using Sentiment Analysis Technique to Study Hotel Service Quality, en: Proceedings of the Annual Hawaii International Conference on System Sciences, art. núm. 6480220, 2013, pp. 3119-3128.

Eisingerich A. y Bell S. Perceived Service Quality and Customer Trust: Does Enhancing Customers' Service Knowledge Matter? Journal of Service Research, volumen 10 (número 3), 2008: 256-268.
Fassnacht M. y Köse I. Consequences of Web-Based Service Quality: Uncovering a Multi-Faceted Chain of Effects. Journal of Interactive Marketing, volumen 21 (número 3), 2007: 35-54.

Glaveli N., Petridou E., Liassides C., Spathis C. Bank Service Quality: Evidence from five Balkan Countries. Managing Service Quality, volumen 16 (número 4), 2006: 380-394.

González M., Comesaña L., Brea J. Assessing Tourist Behavioral Intentions Through Perceived Service Quality and Customer Satisfaction. Journal of Business Research, volumen 60 (número 2), 2007: 153-160.

Hau-Siu C., Lau I., Wing-Chun V., Lo-T.S.Z., Yun H. Service Quality in Restaurant Operations in China: Decision and Experiential Oriented Perspectives. International Journal of Hospitality Management, volumen 26 (número 3), 2007: 698-710.

Ho C. y Lee Y. The Development of an E-Travel Service Quality Scale. Tourism Management, volumen 28 (número 6), 2007: 1434-1449.

Hsieh L., Lin L., LinY. A Service Quality Measurement Architecture for Hot Spring Hotels in Taiwan. Tourism Management, volumen 29 (número 3), 2008: 429-438.

$\mathrm{Hu}$ H., Kandampully J., Juwaheer D. Relationships and Impacts of Service Quality, Perceived Value, Customer Satisfaction, and Image: An Empirical Study. Service Industries Journal, volumen 29 (número 2), 2009: 111-125.

Jasinskas E., Reklaitiene D., Švagždiene B. Evaluation of Service Quality in Fitness Centres. Transformations in Business and Economics, volumen 12 (número 1), 2013: 108-124.

Juárez E. y González O. Mercadotecnia personalizada, en: Megatendencias sociales y su impacto en la identificación de oportunidades estratégicas de negocios, Grupo de desarrollo regional del Tecnológico de Monterrey, primera edición, 2009 [en línea] [fecha de consulta: 13 de mayo de 2013]. Disponible en: http:// www.pmcollege.ca/sitios/ctm/orientacion/Alumnos/Megatendencias/5.\%20Mercadoctecnia\%20personalizada.pdf

Lan J. Research on Gap Model Construction and its Application Method of Comprehensive Sports Center Service Quality. Advanced Science Letters, volumen 19 (número 6), 2013: 1803-1806.

Lee S.Y., Tongzon J.L., Chang Y.-T. Assessing Port Service Quality by Process Component: the Case of Korean and Chinese Ports. International Journal of Shipping and Transport Logistics, volumen 5 (número 2), 2013: 137-154.

Maddern H., Maull R., Smart A., Baker P. Customer Satisfaction and Service Quality in UK Financial Services. International Journal of Operations and Production Management, volumen 27 (número 9), 2007: 999-1019.

Olorunniwo F., Hsu M., Udo G. Service Quality, Customer Satisfaction, and Behavioral Intentions in the Service Factory. Journal of Services Marketing, volumen 20 (número 1), 2006: 59-72.

Oyewole P. The Role of Frequency of Patronage and Service Quality of All-You-Can-Eat Buffet Restaurant: a Perspective of Socio-Economic and Demographic Characteristics of African American Consumers. International Journal of Hospitality Management, volumen 34 (número 1), 2013: 202-213.

Park J., Robertson R., Wu C. Modelling the Impact of Airline Service Quality and Marketing Variables on Passengers Future Behavioural Intentions. Transportation Planning and Technology, volumen 29 (número 5), 2006: 359-381.

Pérez J. Revisión sistemática de literatura en ingeniería, editorial Universidad de Antioquia, Medellín, Colombia, 2012. 
Pérez J. y Jaramillo P. Espacio literario relevante sobre el problema del vendedor viajero (TSP): Contenido, clasificación, métodos y campos de inspiración. Revista Producao, Ahead of print, E-pub 01, febrero de 2013 [en línea] [fecha de consulta: 13 de mayo de 2013]. Disponible en: http://www.scielo.br/ scielo.php? pid=S0103-65132013005000003\&script $=$ sci_ abstract\&tlng=es

Pérez J. y Muños L. Revisión sistemática de literatura sobre la calidad del servicio, Informe técnico inédito, Grupo Gestión de la Calidad, Departamento de Ingeniería Industrial, Universidad de Antioquia, libro 10, tomo 325, partida 180, 20 de abril, 2012, 136p.

Pornpitakpan C., Han J. The Effect of Culture and Salespersons' Retail Service Quality on Impulse Buying. Australasian Marketing Journal, volumen 21 (número 2), 2013: 85-93.

Saura I., Francés D., Contrí G., Blasco M. Logistics Service Quality: A New Way to Loyalty. Industrial Management and Data Systems, volumen 108 (número 5), 2008: 650-668.

Scimago. Análisis de la cobertura de la base de datos Scopus. El Profesional de la Información, volumen 15 (número 2), 2006: 144-145.

Senić V., Marinković V. Patient Care, Satisfaction and Service Quality in Health Care. International Journal of Consumer Studies, volumen 37 (número 3), 2013: 312-319.

Shamdasani P., Mukherjee A. y Malhotra N. Antecedents and Consequences of Service Quality in Consumer Evaluation of Self-Service Internet Technologies. Service Industries Journal, volumen 28 (número 1), 2008: 117-138.

Tsai H. y Lu I. The Evaluation of Service Quality Using Generalized Choquet Integral. Information Sciences, volumen 176 (número 6), 2006: 640-663.

Tseng M. Using the Extension of DEMATEL to Integrate Hotel Service Quality Perceptions into a Cause-Effect Model in Uncertainty. Expert Systems with Applications, volumen 36 (número 5), 2009: 9015-9023.

Tsoukatos E. y Rand G. Path Analysis of Perceived Service Quality, Satisfaction and Loyalty in Greek Insurance. Managing Service Quality, volumen 16 (número 5), 2006: 501-519.
Ueltschy L., Laroche M., Eggert A., Bindl U. Service Quality and Satisfaction: An International Comparison of Professional Services Perceptions. Journal of Services Marketing, volumen 21 (número 6), 2007: 410-423.

Wilkins H., Merrilees B., Herington C. Towards an Understanding of Total Service Quality in Hotels. International Journal of Hospitality Management, volumen 26 (número 4), 2007: 840-853.

Yap K. y Sweeney J. Zone-of-Tolerance Moderates the Service Quality-Outcome Relationship. Journal of Services Marketing, volumen 21 (número 2), 2007: 137-148.

Yen C. y Lu H. Effects of E-Service Quality on Loyalty Intention: An Empirical Study in Online Auction. Managing Service Quality, volumen 18 (número 2), 2008: 127-146.

Yi Y. y Gong T. The Electronic Service Quality Model: the Moderating Effect of Customer Self-Efficacy. Psychology and Marketing, volumen 25 (número 7), 2008: 587-601.

\section{Este artículo se cita:}

\section{Citación estilo Chicago}

Pérez-Rave, Jorge Iván, Leandro Muñoz-Giraldo. Espacio literario relevante sobre la evaluación de la calidad del servicio: países de realización de los estudios, índices de fiabilidad, hipótesis y desafíos. Ingeniería, Investigación y Tecnología, XV, 03 (2014): 479-492.

\section{Citación estilo ISO 690}

Pérez-Rave J.I., Muñoz-Giraldo L. Espacio literario relevante sobre la evaluación de la calidad del servicio: países de realización de los estudios, índices de fiabilidad, hipótesis y desafíos. Ingeniería, Investigación y Tecnología, volumen XV (número 3), julio-septiembre 2014: 479-492.

\section{Semblanza de los autores}

Jorge Iván Pérez-Rave. Ingeniero industrial de la Universidad de Antioquia (Medellín. Colombia). Especialista y magíster en ingeniería de sistemas de la Universidad Nacional de Colombia, sede Medellín. Director del grupo de investigación Gestión de la Calidad. Docente e investigador del Departamento de Ingeniería Industrial de la Universidad de Antioquia desde 2008. Conferencista, consultor, autor de libros y de varios artículos publicados en revistas de carácter nacional e internacional. Ha servido como árbitro en revistas nacionales e internacionales. Entre sus reconocimientos recientes están: Premio “Mejor evaluación del año 2012" otorgado por el Centro de Información Tecnológica, La Serena, Chile. Par evaluador de Colciencias, 2012-2014 y Premio a la investigación docente 2011, Facultad de Ingeniería de la Universidad de Antioquia.

Leandro Muñoz-Giraldo. Ingeniero industrial de la Universidad de Antioquia, integrante del grupo de investigación Gestión de la Calidad en la línea de calidad del servicio. Analista en la Unidad de Consultoría basada en investigación. 